\title{
Analysis of DAC MAC RBAC Access Control based Models for Security
}

\author{
Bokefode Jayant. D. \\ Research Scholar, \\ Sinhyad College of \\ Engineering, korti, \\ Pandharpur,Solapur University, \\ INDIA
}

\author{
Ubale Swapnaja A. \\ Assistant Professor \\ Sinhgad College of \\ Engineering, korti, Pandharpur, \\ Solapur University,INDIA
}

\author{
Apte Sulabha S., PhD \\ HOD, Department of Computer \\ Science and Engineering. \\ Walchand Institute of \\ Technology, \\ Solapur University, INDIA
}

\author{
Modani Dattatray G. \\ Assistant Professor \\ Gharda Institute of Technology, \\ Lavel, Chiplun, \\ Mumbai University, INDIA
}

\begin{abstract}
Access Control is the process or mechanism for giving the authority to access the specific resources, applications and system. Access control defines a set of conditions or criteria to access the system and its resources. There are three main accesses Control model first is Mandatory access control model, second is Discretionary access control model and third is Role based access control models. In Mandatory access control models, the user's roles are allotted according to the system administrator wishes. In this, end users do not have authority to set any access control policies on files therefore it is the most restrictive access control method. It is useful in a highly secured environment. For example military, research centers. In Discretionary access control model, the end users have complete authority to assign any rights to objects. But giving all control to the user over the files is too dangerous because if an attacker got the control over the account then the attacker will have complete authority on the access. In Role based model creates different authorities permissions by assigning access rights to specific roles or jobs within the company then role based access control assigns these roles to users. It is effectively implemented in an organization because files and resources are assigned according to the roles. Assigning roles to the user was done by the system administrator. In this, Roles are assigned affected to each resource. For example, roles can decide a resource to be used at certain times of the day.
\end{abstract}

\section{Keywords}

Access Controls, Mandatory Access control (MAC), Discretionary access control (DAC), Role based access control (RBAC).

\section{INTRODUCTION}

To develop any organizational system, information management system and any application we need to protect data and resources against unauthorized access and unauthorized modifications while at the same time ensuring their availability. Therefore it is necessary to ensure that the entire access request should be made by the authorized user. To develop a access control system, requires the regulations and different rules according to which access is to be controlled. The development process is carried out with a different multiphase approaches. These approaches based on the following concepts [1] [2][3].

Security policy: Security policies are nothing but set of rules defining who is authorized to access what and under which conditions, and the criteria under which such authorization is given or cancelled.

Security model: It gives implementation of the access control security policies and it's working.

Security mechanism: It defines the different low level (software and hardware) functions that implement the controls described by the policy and stated in the model.

In real world situations have number of complex policies, in this access decisions depend on the rule. According to the different application rules are coming, for example, from organizational regulations, practices and government laws. To develop the access control system need to ensure that the availability of the resources, confidentiality and integrity of the data.

There are three main types of access control policies.

Mandatory access control (MAC), in this security policy users do not have the authority to override the policies and it totally controlled centrally by the security policy administrator. The security policy administrator defines the usage of resources and their access policy, which cannot be overridden by the end users, and the policy, will decide who has authority to access the particular programs and files. MAC is mostly used in a system where priority is based on confidentiality.

Discretionary access control (DAC), this policy Contrast with Mandatory Access Control (MAC) which is determined by the system administrator while DAC policies are determined by the end user with permission. In DAC, user has the complete authority over the all resources it owns and also determines the permissions for other users who have those resources and programs. 
Role-based access control (RBAC), this policy is very simple to use. In RBAC roles are assigned by the system administrator statically. In which access is controlled depending on the roles that the users have in a system. (RBAC) is mostly used to control the access to computer or network resources depending on the roles of individual users within an organization. In this literature we describe the different access control policies and models that have been proposed by the researchers, also finding the current status of access control systems and their low level implementation in terms of security mechanisms. This review gives the idea of different access control policies to develop the access control systems and gives the comparison of the different security policies and their mechanisms.

\section{ACCESS CONTROL POLICIES AND MODELS}

There are three main types of access control policies.

\subsection{Discretionary Access Control (DAC)}

DAC was developed to implement Access Control Matrices defined by Lampson in his paper on system protection [4]. Discretionary policies defines access control based on the identity of the requestors and explicit access rules that determines who can, or cannot, execute particular actions on particular resources. In DAC users can be given the authority to other users to access the resources, where assigning and granting the privileges is done by an administrative policy. Different types of DAC policies and models have been proposed in the literature.

\subsubsection{The access matrix model}

It provides a simple framework for implementing the discretionary access control. It is proposed by Lampson [5] for providing protection against the unauthorized access to the resources within the operating systems and later it is refined by Graham and Denning [6], the model was developed by Harrison, Ruzzo, and Ullmann (HRU model) [7], to minimize the complexity of access control policy. This model is called as access matrix. Access matrix holds the authorization state at a given time in the system. It provides the abstract representation of protection systems.

\begin{tabular}{c|c|c|c|c|}
\multicolumn{1}{c}{} & \multicolumn{1}{c}{ File 1 } & \multicolumn{1}{c}{ File2 } & \multicolumn{1}{c|}{ File3 } & Program-1 \\
\cline { 2 - 5 } Jack & $\begin{array}{c}\text { own } \\
\text { read } \\
\text { write }\end{array}$ & $\begin{array}{c}\text { read } \\
\text { write }\end{array}$ & execute \\
\cline { 2 - 5 } Tom & read & $\begin{array}{c}\text { read } \\
\text { write }\end{array}$ & \\
\cline { 2 - 5 } Kate & & read & $\begin{array}{c}\text { execute } \\
\text { read }\end{array}$ \\
\cline { 2 - 5 } & & &
\end{tabular}

Fig 1: An example of access matrix

To design an access control system a first step is the identification of the objects which we have to be protected and the executing access request and different activities to objects, and the actions that can be executed on the objects and that must be controlled. For example, in the operating systems, objects can be any programs, directories or files.
The authorization state in the access matrix model is defined by a triple $(\mathrm{S}, \mathrm{O}, \mathrm{A})$, where $\mathrm{S}$ is the set of subjects, who can have access liberties; $\mathrm{O}$ is the set of objects, on which access rights can be exercised (subjects may be considered as objects, in which case $S \subseteq \mathrm{O}$ ); and A is the access matrix, In this rows represents the subjects, columns represents the objects, and entry $\mathrm{A}[\mathrm{s}, \mathrm{o}]$ reports the access rights of $\mathrm{s}$ on $\mathrm{o}$. The access control model simply provides a framework where authorizations can be specified, the model can contain different access rights or privileges. For example, read, write, and execute actions can be considered with ownership (i.e., property of objects by subjects), and control (to model fatherchildren relationships between processes) privileges. We can change the state of a system by executing different commands that can execute primitive operations on the authorization state with some conditions.

\subsubsection{Disadvantages of DAC}

Global policy: DAC allows user to decide access control policies on their resources and these policies are global policies and therefore DAC has trouble to ensure consistency.

Malicious software/programs: DAC is vulnerable from processes because it executing malicious programs. If it execute the malicious programs exploiting the authorizations of a particular user on behalf of whom they are executing. For instance, Trojan Horses.

Information flow: Once the particular information is acquired by a process, and then DAC do not have any control on the flow of information. Information can be copied from one object to another; therefore it is possible to access a copy even if the owner does not provide the access to the original copy.

\subsection{Mandatory Access Control (MAC)}

In MAC users do not have the authority to override the policies and it totally controlled centrally by the security policy administrator.

MAC is a system-wide policy which defines who is allowed to have access; individual user cannot change that access rules. It totally relies on the central system.MAC policies are defined by the system administrator, and it is strictly enforced by the OS or security kernel.

Examples:

According to law, court can access driving records without the owners' permission. MAC mechanisms have been tightly coupled to a few security models and it is mostly used in a system where priority is based on confidentiality. For example Trusted Solaris, TrustedBSD, SELinux etc.)

MAC can be classified in to following types.

1. Multilevel Security

2. Multilateral Security

\subsubsection{Multilevel Security}

In this, information and users are classified into different levels according to their sensitivity and trust. It will be classified into Confidential, Secret and Top Secret. This defines different levels such as clearance level, classification level and security level. 
- Clearance level indicates how much trust or rights given to a person with some clearance. The trust or given rights indicates the highest level of classified information handled by the users or device.

- Classification level indicates the level of sensitivity to be given for a particular resources or information. For instance, the level may indicate the degree of damage the country if the information is disclosed to an enemy.

- Security level is a general term for the classification level or clearance level.

In government and military facilities, MAC performs classification and then assigns a label to each file system object. According to the level of security it include confidential, secret and top secret. When a user or device tries to access particular files or resources, the OS or security kernel determine whether access will be granted or not. MAC requires continuous monitoring and careful planning to keep all resource objects' and users' classifications up to date.

\subsubsection{The Bell-LaPadula Security Policy Model}

It is proposed by David Bell and Len Lapadula in 1973, to provide security for time-sharing mainframe systems. This model also called as MLS model [10]. This model dealt with confidentiality. In this model, two types of security label are assigned to subjects and objects based on the simple security property and *-property to verifiably ensure military classification policies that restrict information flow from more secure classification levels to less secure levels. Also referred as No read up and No write down[8][9].

Simple security property: It states that process labeled with higher classification cannot access or read information or resources.

That is, Subject $\mathrm{A}$ is allowed to read object $\mathrm{O}$ only if class (O) $\leq$ class (A).

*-property: It does not allow processes from writing to a lower classification. That is, Subject A is allowed to write object $\mathrm{O}$ only if class $(\mathrm{A}) \geq \operatorname{class}(\mathrm{O})$.

These two properties are enhanced by the tranquility property, which is described in two types: strong and weak. In strong tranquility property, we cannot change the labels during system operation. In weak tranquility property, however, we can change the labels during system operation without violating defined security policies [11].

The main advantages of the weak tranquility property are that it give rights in the lowest security session while starting a user session, have its classification level reduced and all objects created.

\subsubsection{The Biba model}

The above mandatory access control model only dealt with confidentiality of the information but not with the integrity of information. To provide the integrity of information new mandatory model is designed by Ken Biba[12], which controls the flow of information and does not allow subjects to modify the information directly.

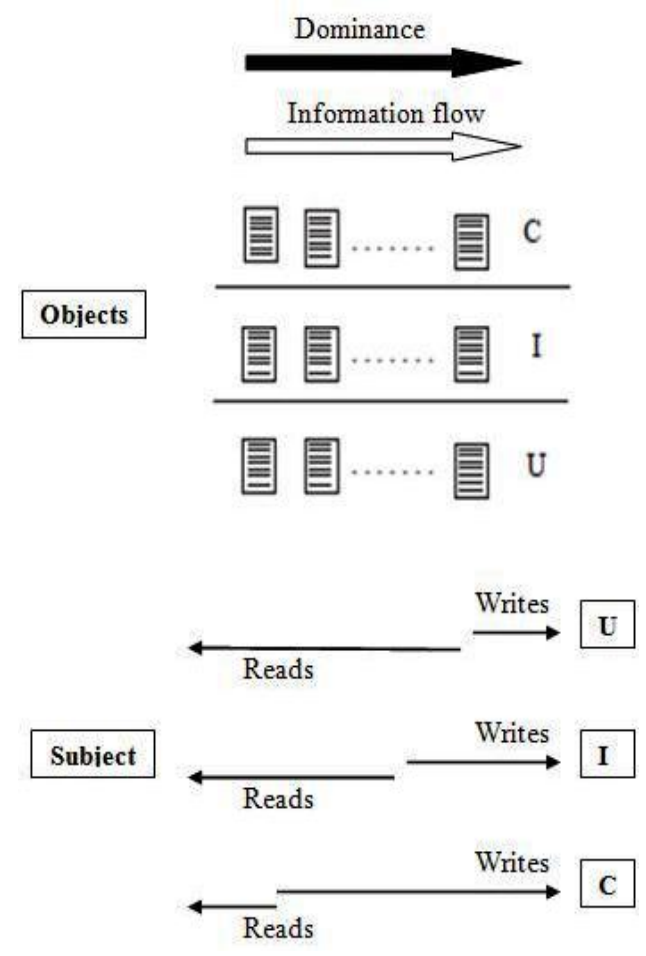

Fig 2: Integrity level for information flow

Integrity levels can be defined as follows: Crucial (C), Important (I), and Unknown (U).The integrity level describes the user's responsibility for modifying, inserting, or deleting information. Object replicate both the degree of trust and the damage that happen due to unauthorized modifications of the information. Access control is described according to the following two principles:

No-read-down In this, subject is allowed to read an object only if the object control the access class of the subject.

No-write-up In this, subject is allowed to write an object only if the subject control the access class of the object.

Simple Integrity Property: In which low integrity subject cannot have the rights to write or modify high integrity data.

*-Property: In which high integrity subject cannot have the rights to read low integrity data.

Biba also proposed alternative criteria for safeguarding integrity, by providing more vibrant controls. These contain the following two policies.

Low-water mark for subjects it control write operations according to the no-write up principle. No restriction is forced on read operations.

Low-water mark for objects it controls the read operations according to the no-read down principle. No restriction is forced on write operations.

\subsubsection{Multilateral Security}

As we know that, mandatory policies provide better security than discretionary policies, therefore it could be used to control indirect information flows. Different policies are proposed as a mixture of mandatory flow control and discretionary authorizations. Here we describe some of them. 


\subsubsection{The Chinese wall policy}

The Chinese Wall [13] policy was proposed by Brewer and Nash to define access rules in a consultancy business where business analysts have to ensure that no conflicts will be occurred in the interest of clients while dealing with multiple clients.The main goal is to control the information flows, due to which conflict will be occurred in a interest of individual consultants (e.g., an individual consultant does not have the information of two companies). In the proposed model, the data objects are organized hierarchically as follows:

Basic objects are the separate items of information for example- files and each concerning to a single corporation.

Company datasets define the collection of objects that related to a same corporation;

Conflict of interest classes (CoI) define the separate company datasets in which conflicts occurred.

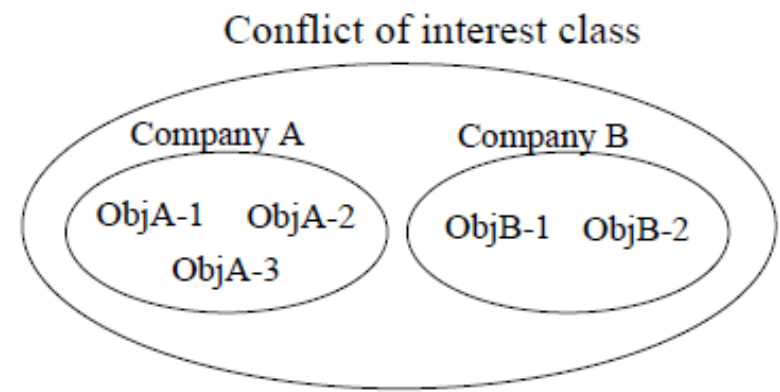

Fig 3: An example of object organization

Figure 3 gives an example of data organization where five objects of two different corporations, namely A and B, are maintained.

According to the object organization as above, the Chinese wall policy controls the access according to the following two properties [13]:

Simple security rule (read rule) a subject $\mathbf{S}$ can provide read access to an object $\mathbf{O}$ only if:

1. The object $\mathbf{O}$ belongs to the same company datasets that is," In the Wall",

2. It belongs to a totally different conflict of interest class.

The simple security rule or read rule blocks the direct information leakages that can be caused due to a single user.

*-property (Write rule) a subject $\mathbf{S}$ can provide write access to an object $\mathbf{O}$ only if:

1. Subject $\mathrm{S}$ can read $\mathrm{O}$ as per to the Read Rule.

2. Object not belongs to a different company dataset (i.e., not O's company dataset) can be read.

This blocks the indirect information leakages that can be caused due to the collusion between two or more users.

\subsubsection{Disadvantages of MAC}

MAC models put restrictions on user access that, and according to security policies, does not allow for dynamic alteration.

MAC needs to place the operating system and associated utilities outside the access control frame work.
MAC requires predetermined planning to implement it effectively. After implementing it needs a high system management because due to constantly update object and account labels to collect new data.

\subsection{Role-based access control (RBAC)}

For providing access rights to user it is important to know the user's responsibilities assigned by the organization. But in the DAC user rights of data plays an important part, are not a good and in MAC, users have to take security clearances and objects need security classifications. RBAC try to reduce the gap by combining the forced organizational constraints with flexibility of explicit authorizations [14].

RBAC mostly used for controlling the access to computer resources. RBAC is very useful method for controlling what type of information users can utilize on the computer, the programs that the users execute, and the changes that the users can make. In RBAC roles for users are assigned statically, which is not used in dynamic environment. It is more difficult to change the access rights of the user without changing the specified roles of the user. RBAC is mostly preferable access control model for the local domain. Due to the static role assignment it does not have complexity. Therefore it needs the low attention for maintenance [15][16].

Role is nothing but the abstractions of the user behavior and their assigned duties. These are used to assign system resources to the departments and their respective members. To provide the accessing control with security in the particular software systems it will be the beneficial to use role concept. It also reduces the cost of authority management [17].

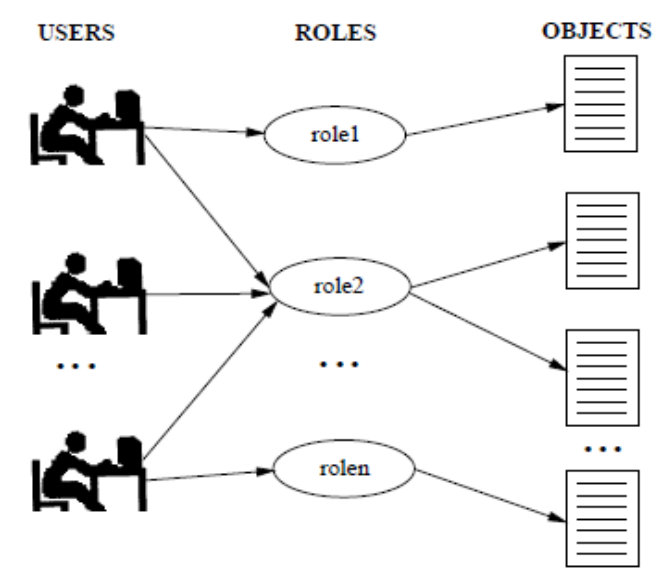

Fig 4: Role-based access control

Essentially, in role based access control policies need to identify the roles in the system, a role can be defined as a set of responsibilities and actions associated with a particular working activity. In an Access control security model, a role is considered as a job related access rights which can be given to the authorized users within an organization. It allows authorized user to achieve its associated responsibilities.

In respect to the RBAC model we describe two types of subjects: the users that related to the system and the transactions which are executed on behalf of those users. Users can access particular objects by executing transactions on that object. A transaction can be referred as a set of executable operations which causes consumption of a system resource [16]. For example, In a bank Tellers are allow to execute a deposit and withdraw transaction, for that it 
requiring read and write access to the specific fields within account. An account supervisor has same or more rights to perform correction transactions.

The system protection is based on the permission that describes a given access right to a particular object or set of objects. In RBAC model we are dealt with unauthorized access to the computer system resources and data [29].

Since we have consider only the access rights that users required to execute a particular transaction on a particular object from the defined set of objects.

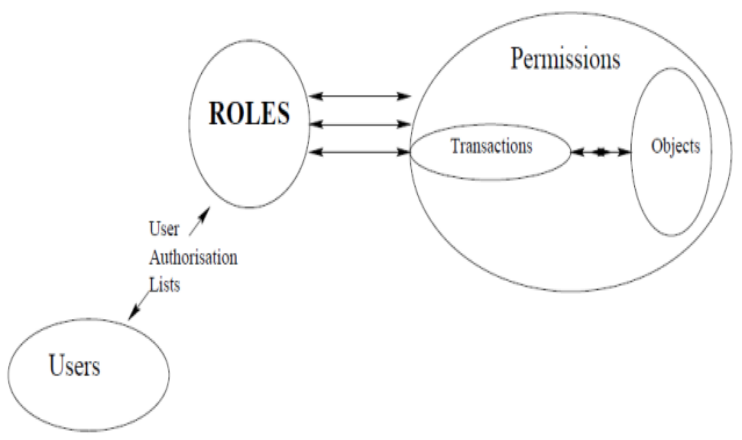

Fig 5: User-Role-Permission Mapping

A permission $\mathrm{p}$ is a pair < trans, objset >, where trans represents the transaction that executes on the set of objects that is objset .Consider $\mathbf{P}$ indicate the universal set of permissions, Trans indicate the universal set of transactions, and Obj indicates the set of objects. We can define the association between permission/transaction and permission/object with the following functions.

Trans $\mathrm{P}(\mathrm{p}): \mathrm{P} \rightarrow \mathrm{Tr}$, It gives the associated transaction to the specified permission $\mathrm{p}$.

$\mathrm{ObP}(\mathrm{p}): \mathrm{P} \rightarrow 2^{\mathrm{Obj}}$,It gives the associated set of objects to the specified permission $\mathrm{p}$.

A role is created by collecting permissions according to the functional and logical requirements to this role should represent. Each role has name associated with this and it uniquely identifies this role in the system.

A role $r$ is a pair of $<\mathrm{rn}$, pset $>$, where $\mathrm{rn}$ indicates the role name and pset indicates the set of role permissions.

The mapping between roles and permissions can be defined with the following function:

$\mathrm{PR}(\mathrm{r}): \mathrm{R} \rightarrow 2^{\mathrm{P}}$, It gives the associated set of permissions to the specified role $r$. Here $R$ indicates the universal set of roles.

While allocating permissions to roles it is need to ensure the principle of least privilege that is each role should have only required rights for its functional requirements.

\subsubsection{Advantages of RBAC}

The role-based access control has the number of advantages. Some of these are described below.

\section{Authorization management:}

Role-based policies provide logical independence in specifying user authorizations. The user authorizations task can be broken down in to two parts: i) assigning roles to the particular users, and ii) assigning objects to roles. This make simpler to manage the security policy: For example, when a new user joins the organization, the administrator of the system needs to grant particular roles as per the job responsibilities; If a user's job responsibilities get changed, then the administrator needs to change the roles associated with that user; when a new task or program is added to the security system, then the administrator needs to decide which roles are provided to execute it.

\section{Hierarchical roles:}

In many applications or organizations have hierarchy of roles, it is based on the principles of generalization and specialization. Figure 21 demonstrate an example of role hierarchy:

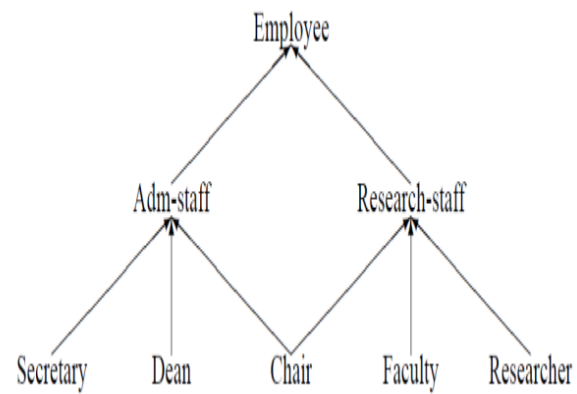

Fig 6: An example of role hierarchy

The role hierarchy can be used to describe the authorization. For example, authorizations can be granted to roles according to the specializations (e.g., the secretary has all accesses granted to adm-staff). Authorization implication can be compulsory on role assignments, by allowing users to use generalizations (e.g., If a user has rights to activate secretary will also be have rights to activate role adm-staff)[19]. The hierarchy needs to be exploited in [18][20] terms of administrative privileges: As per hierarchy of organizational roles, an additional hierarchy should be defined for administrative roles; administrative role can have rights over the role hierarchy.

\section{Least privilege}

Roles defines the least privilege that user required to perform the particular task. Those Users are authorized to powerful roles do not need to use them until those rights are actually needed. This minimizes the damage happens due to the unintended errors [21].

\section{Separation of duties}

This principle describe that no user should have more rights so he can misuse it. For instance, the person who authorized a paycheck and who can prepare them should not be the same person. Separation of duties can be done either statically or dynamically. In statically, it can be done by defining conflicting roles. In dynamically, it can be done by providing the control at the access time. For example, separation of duties of two-person rules [22].

\section{Constraints enforcement}

Roles give the specification and enforcement that required for protection that real world policies may need to define. For example, cardinality constraints specified that the number of roles allow executing on a given privilege and the number of 
users is restricted to activate a role. The constraints can be a dynamic. It can be forced on roles activation instead of their assignment. We can implement Role Based Access control in numerous domains such as web enhancement, controlling systems to providing access rights to the authorized person only [32][30].

\subsubsection{Disadvantages of RBAC}

In RBAC model, there is still some work to be done to cover up all the requirements which may represent the real world scenario.

Defining the roles in a different context is difficult and it may result into large role definition. Sometimes it produces more roles than users.

Now days, require fine grained results but RBAC not gives fine grained results [23].

RBAC assigns the roles statically to its user, which is not preferred in dynamic environment. It is difficult to implement when the environment is dynamic and distributed. Due to this it is more difficult to change the access rights of the user without changing the role of that user. Therefore RBAC not provide support for dynamic attributes such as time of the day on which the user permission is determined.

It maintains the relation between users and its roles. It also maintains the relation between permissions and roles. Therefore to implement the RBAC model roles must be assigned in advance and it is not possible to change access rights without altering the roles.

\section{DYNAMIC TYPE ACCESS CONTROL}

Dynamic type Access Control (DTAC) is extended from Type Enforcement (TE). The type enforcement principle is more flexible in which columns in the access control matrix are changed to the type and objects are assigned to types. [25] The Domain Type Enforcement (DTE) is an extension of Type Enforcement (TE). In this subjects are replaced with domains and access matrix is transformed in to the domain definition table (DDT) in which rows represent domains and columns represent types. DTAC stretched upon this to include RBAC type administrative controls. [24] It is state that DTE models can apply the Bell-LaPadula confidentiality model and some robust integrity features in DAC and RBAC.

\section{ATTRIBUTE BASED ACCESS CONTROL MODEL (ABAC)}

In $\mathrm{ABAC}$, permissions to access the objects are not directly given to the subject. It uses attributes of the subjects and objects to provide authorizations. For subjects, we consider static attributes like a subject's name, or designitation or role in an organization and dynamic attributes like age, current location or an acquired subscription for a digital library. For objects, we consider metadata properties such as the subject of a document can be used. The functionality of ABAC model is shown in figure 7. Permissions contain the combination of an object descriptor and operations, where Object Descriptor is a combination of a set of attributes and conditions. Operation describes the instructions denoted by the descriptor which is executed on the objects. Access rights can be defined between a subject descriptor and permission. Using descriptors we can dynamically assign permissions to subjects and objects. $\mathrm{ABAC}$ uses subject, object, and their environment attributes. Before using these attributes for making access control decision the attribute document is checked for the integrity and validated [27][31].

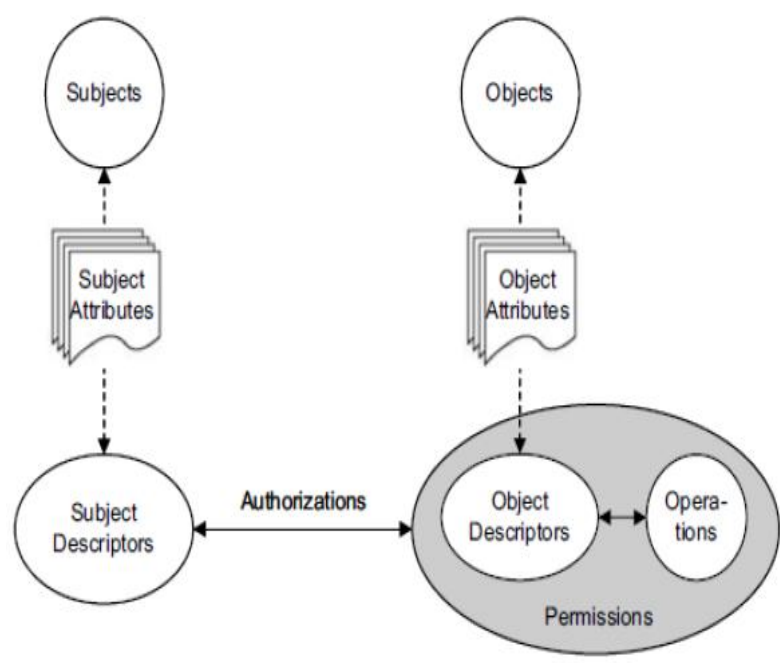

Fig 7: Overview of the ABAC model [28]

In $\mathrm{ABAC}$ permissions are depending on the combination of the subjects and object attribute values.

\subsection{Advantages of $A B A C$}

$\mathrm{ABAC}$ solves the user role assignment problem which present in RBAC and instead of focusing the roles it focuses on the attributes of a user to assigns the access rights.

ABAC gives higher flexibility in a distributed, open, sharable and dynamic environment where the numbers of users are very high. Therefore ABAC is very flexible model for the administrating purpose and it work very well than RBAC [26].

ABAC also support for the global agreement for attributes so that attributes which are provided in one domain can be forward to the other domain at the point of domain to domain interaction.

ABAC also used for web administrator to improve the web site structure with Web Enhancement Model expressed in [30].

It provide central storage for user attributes, it increase the interoperability and sharing between several service providers to decide the user rights.

\subsection{Disadvantages of ABAC}

Due the heterogeneity of user information complexity is increased, therefore to solve this it require the central database having all attributes in same format

On the other hand, if the multiple organizations decide common set of standardized attributes, this would raise the problem of low expressiveness for representing the subjects and objects, therefore it losing the advantages of the flexible and dynamic ABAC functionality [28].

\section{CONCLUSION \& FUTURE WORK}

In this paper we have studied MAC, DAC and RBAC access control models and there different implementations. Also we have listed the advantages and disadvantages of these models. Still some work needs to be done on interpreting policies into acceptable model to provide efficient and accurate management of these models. Developing a new model such as, Dynamic Typed Access Control (DTAC) and Attribute- 
Based Access Control (ABAC) to overcome the problem of RBAC. Operating systems are also likely to expand support for additional access control models both internally and with Pluggable Policy Modules to allow users and administrators more comprehensive and user-friendly ways to secure systems.

In future we will integrate the two models $\mathrm{RBAC} \& \mathrm{ABAC}$ in such a way that can overcome the existing problems with RBAC \& ABAC and can get a fine grained access control model which is highly demandable in shareable, open and changing environment.

Future work in this area is likely to be focused on the production of Role-Based Access Control models for community cyber security. Oracle also supports RBAC as part of their database management access controls to support role based access control.

\section{REFERENCES}

[1] D.D. Clark and D.R. Wilson. A Comparison of commercial and Military Computer Security Policies. In IEEE Symposium on Computer Security and Privacy, April 1987.

[2] A. Aho, J. Hoperoft, and J. Ullman. The Design and Analysis of Computer Algorithms.Addison-Wesley, 1974.

[3] B.W. Lampson. Protection. In 5th Princeton Symposium on Information Science and Systems, pages 437-443, 1971. Reprinted in ACM Operating Systems Review 8(1):18-24, 1974.

[4] B. W. Lampson. Protection. ACM SIGOPS Operating System Review, 8(1):18-24, January 1974.

[5] G.S. Graham and P.J. Denning. Protection - principles and practice. In AFIPS Press, editor, Proc. Spring Jt. Computer Conference, volume 40, pages 417-429, Montvale, N.J., 1972.

[6] M.H. Harrison, W.L. Ruzzo, and J.D. Ullman. Protection in operating systems. Communications of the ACM, 19(8):461-471, 1976

[7] R.S. Sandhu. The typed access matrix model. In Proc. of 1992 IEEE Symposium on Security and Privacy, pages 122-136, Oakland, CA, May 1992.

[8] D.E. Bell. Secure computer systems: A refinement of the mathematical model. Technical Report ESD-TR-278, vol. 3, The Mitre Corp., Bedford, MA, 1973.

[9] D.E. Bell and L.J. LaPadula. Secure computer system: Unified exposition and multics interpretation.Technical Report ESD-TR-278, vol. 4, The Mitre Corp., Bedford, MA, 1973.

[10] D.E. Bell and L.J. LaPadula. Secure computer systems:Mathematical foundations. Technical Report ESD-TR-278, vol. 1, The Mitre Corp., Bedford, MA, 1973.

[11] L.J. LaPadula and D.E. Bell. Secure computer systems: A mathematical model. Technical Report ESD-TR-278, vol. 2, The Mitre Corp., Bedford, MA, 1973.
[12] K.J. Biba. Integrity considerations for secure computer systems. Technical Report TR-3153,The Mitre Corporation, Bedford, MA, April 1977.

[13] D.F.C. Brewer and M.J. Nash. The Chinese Wall security policy. In Proc. IEEE Symposium on Security and Privacy, pages 215-228, Oakland, CA, 1989.

[14] H. L. F. Ravi S. Sandhu, Edward J. Coyne and C. E. Youman. Role-based access control models. IEEE Computer, 29(2):38-47, February 1996.

[15] R. Sandhu. The next generation of access control models: Do we need them and what should they be? In SACMAT'01, page 53. SACMAT, May 2001

[16] D. Ferraiolo and R. Kuhn. Role-based access controls. In Proc. of the 15th NIST-NCSC Naional Computer Security Conference, pages 554-563, Baltimore, MD, October 1992.

[17] YAO Zhilin, LI Bing and LIU Shufen, "Role Based Collaboration Authorizing by Using Ontology", Chinese Journal of Electronics Vol.20, No.3, July 2011.

[18] R. Sandhu and Q. Munawer. The ARBAC99 model for administration of roles. In Proc. Of the 15th Annual Computer Security Applications Conference, Phoenix, Arizona, December 1999.

[19] R. Sandhu, E.J. Coyne, H.L. Feinstein, C.E. Youman. The ARBAC97 Model for Role-Based Administration of Roles. In Proceedings of 2nd ACM Work-shop on Role Based Access Control, 1997.

[20] R. Sandhu, Q. Munawer. The RRA97 Model for RoleBased Administration of Role Hierarchies. In Proceedings of 3rd ACM Workshop on Role Based Access Control, 1998.

[21] A. Zakinthinos. A Least Privilege Mechanism for User Processes. Masters Thesis, Department of Computer Science, University of Toronto, 1993

[22] Ravi S. Sandhu. Transaction control expressions for separation of duties. In Fourth Annual Computer Security Application Conference, pages 282-286, Orlando, FL, December 1988

[23] Bernard Stepien, Stan Matwin, Amy Felty, "Advantages of a Non-Technical XACML Notation in Role-Based Models", 2011 Ninth Annual International Conference on Privacy, Security and Trust.

[24] J. A. Solworth and R. H. Sload. Security property based administrative controls. 2005

[25] R. Watson. Statement for the sacmat 2001 panel. In SACMAT'01, page 149. SACMAT, May 2001.

[26] Bernard Stepien, Stan Matwin, Amy Felty, "Advantages of a Non-Technical XACML Notation in Role-Based Models”, 2011 Ninth Annual International Conference on Privacy, Security and Trust. 
[27] Gerald Stermsek, Mark Stremmbeck, Gustaf Neumann, "Using Subject- and Object-specific Attrubutes for Access Control in Web-based Knowledge Management System”.

[28] Torsten Priebe, Wolfgang Dobmeier, Christian Schläger, Nora Kamprath, "Supporting Attribute-based Access Control in Authorization and Authentication Infrastructures with Ontologies", First International Conference on Availability, Reliability and Security (ARES 2006), Vienna, Austria, April 2006.

[29] Prof. S.A.Ubale and Dr. S.S. Apte, "Study and Implementation of Code Access Security with .Net Framework for Windows Operating System", International Journal of Computer Engineering \& Technology (IJCET), Volume 3, Issue 3, 2012, pp. 426 434, ISSN Print: 0976 - 6367, ISSN Online: 0976 - 6375

[30] Bokefode J.D, Ubale S. A, Modani D. G, Bhandare P.S. "Enhancing the web site structure to provide easy traversal on a website with minimum changes to its structure ", International Journal of Computer
Engineering \& Technology (IJCET), Volume 5, Issue 1, January (2014),ISSN Print: 0976 - 6367, ISSN Online: $0976-6375$.

[31] Prof. S. A. Ubale, Dr. S. S. Apte, "Comparison of ACL Based Security Models for securing resources for Windows operating system ", IJSHRE Volume 2 Issue 6 Page No 63

[32] Bhandare P.S, Bokefode J.D, Bhise A. S, More P. B, "Analysis of Electrocardiograph using Perceptron Feed Forward Neural Network" International Journal of Computer Applications (0975 - 8887)Volume 90 - No 1, March 2014.

[33] Sonu Verma, Manjeet Singh, Suresh Kumar, "Comparative analysis of Role Base and Attribute Base Access Control Model in Semantic Web", International Journal of Computer Applications (0975 - 8887) Volume 46- No.18, May 2012. 